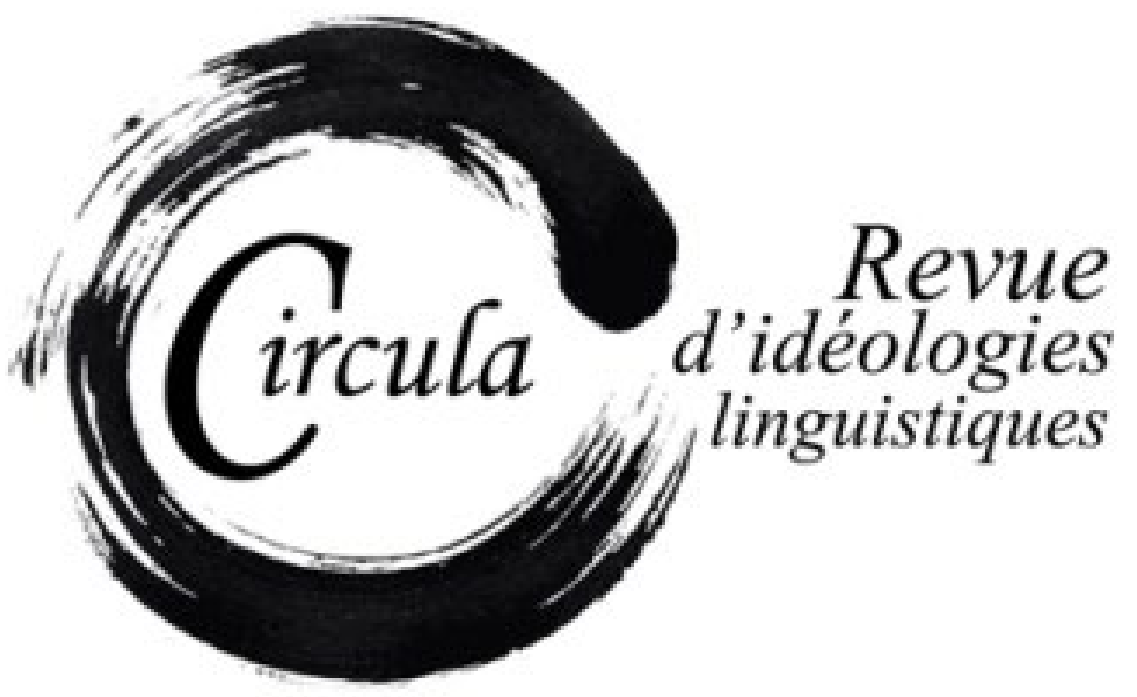

TITRE: LA CICALATA E LA SUA CRITICA: VARIETÀ DI VALUTAZIONI LINGUISTICHE SU UN GENERE LETTERARIO tra SetTe e OtTocento

Auteur(s): FABIO Ruggiano, UniVersitÀ di Messina

Revue: CirCula, NUMÉRO 4

PAGES: $1-21$

ISSN: 2369-6761

DiRecteurs: Wim Remysen et SABIne SchWARZE

URI: HTTP://HDL.HANDLE.NET/11143/10174

DOI: $10.17118 / 11143 / 10174$ 


\section{La cicalata e la sua critica: varietà di valutazioni linguistiche su un genere letterario tra Sette e Ottocento}

Fabio Ruggiano, Università di Messina fruggiano@unime.it

Riassunto: Questo contributo si propone di mostrare come l'immagine ideale della lingua italiana si sia evoluta tra il Settecento e l'Ottocento, seguendo il cambiamento dei quadri culturali di riferimento, i rapporti di forza tra classicismo e modelli linguistico-letterari emergenti e, su un piano più generale, il progressivo rafforzamento del sentimento di identità nazionale. Questa evoluzione sarà seguita ripercorrendo i giudizi di intellettuali diversi - e risalendo ai principi ispiratori di quei giudizi - espressi su alcune delle principali riviste letterarie italiane tra Settecento e Ottocento (Frusta letteraria, /l Caffè, /l Conciliatore, /l Poligrafo, Antologia) a proposito del classicismo linguistico e stilistico dell'Accademia della Crusca. In particolare, le posizioni ideologiche sulla lingua in favore o contro la Crusca saranno valutate in relazione ai giudizi espressi non sul Vocabolario, ma sul genere letterario della cicalata, tanto amato dagli accademici, baluardo degli atteggiamenti linguistici più fiorentinocentrici e della pratica letteraria più avulsa dalla attualità politica, sociale, culturale.

Parole chiave: cicalata; Accademia della Crusca; riviste letterarie; classicismo; illuminismo; romanticismo

Abstract: This article attempts to show how the ideal image of the Italian language has evolved between the XVIII and XIX century, following the change of cultural frameworks of reference, the balance of forces between classicism and emerging linguistic literary models and, more generally, the gradual strengthening of the national identity feelings. This evolution will be followed retracing the opinions of several intellectuals - and going back to the principles of those opinions - expressed on some of the main Italian literary magazines between the XVIII and XIX centuries (Frusta letteraria, Il Caffè, /l Conciliatore, /l Poligrafo, Antologia) about the linguistic and stylistic classicism of the Accademia della Crusca. In particular, the ideological positions about language in favor or against the Crusca will be evaluated in relation to opinions expressed not on the Vocabolario, but on the literary genre known as cicalata, so loved by the academics, bulwark of the most Florentine-centered linguistic attitudes and of a literary practice completely divorced from the current political, social, cultural world.

Keywords: cicalata; Accademia della Crusca; literary magazines; classicism; enlightenment, romanticism 


\section{Introduzione}

Il tipo di componimento noto come cicalata fu un cardine della vita dell'Accademia della Crusca fin dalla svolta impressa da Lionardo Salviati nel 1582 ed ebbe, per i cruscanti, lo scopo di esaltare la potenza espressiva della lingua fiorentina non solamente nella letteratura alta e nella trattatistica, ma anche nella prosa burlesca e di intrattenimento. Per quasi tre secoli gli accademici si cimentarono in questi componimenti, così descritti da Anton Maria Salvini (1715/1734: prefazione):

Oltre a questi serii Esercizj non mancano i giocosi, come sono le Cicalate, fatte in occasione di pubblico Stravizzo, che così si nomina il Convito Accademico; e questa è una sorta di Lezione in burla, che si recita dopo cena, dalle Leggi dell'Accademia strettamente ingiunta, e ad ogni Accademico comandata, non solamente per ristorar gli animi dalle studiose fatiche con amena, ed erudita ricreazione, come anche per impiegare il bel talento di nostro ricco linguaggio, che siccome le gravi materie sostiene, così alle giocose per la sua proprietà, ed acume è acconcissimo.

I principi, le caratteristiche e gli scopi del genere sono spiegati più eloquentemente, insieme ai pregi della nostra Toscana lingua, nella anonima prefazione di Prose fiorentine (1723: III-V)1:

Pregio grandissimo delle nobili, e leggiadre favelle da' giusti stimatori delle cose viene a buona equità riputato, che sieno elleno di così copiose ricchezze abbondevolmente fornite, che ad ogni diversa maniera d'argomenti, che tuttora in quelle convien trattare, sieno dicevoli, e proprie, e in bella guisa adattate. Questo pregio, e questa singolar lode, si debbe certamente alla nostra Toscana lingua, la quale è cosi sonora, vaga, grave, forte, maestosa, gentile, tenera, armoniosa, soave, che qualunque sorta di componimenti possono in essa trattarsi, e forza riceverne, e vaghezza, e splendore. [...] Quindi è che per proseguire questa, per mio avviso, lodevole inchiesta, nel presente Sesto Volume della Prima Parte, si è voluto racchiudere una maniera di componimento così particolare di questa favella, che non ve n'è stata forse alcun altra finora, che uno cosi fatto ne abbia avuto giammai. Fu questo ritrovato, ed alla sua intera perfezione felicemente condotto, da quegli avventurosi ingegni celebri fondatori dell'Accademia della Crusca, che bramosi d'ingrandire, e far ricco, e render sempre più grazioso, e adorno l'idioma loro, saggiamente divisarono, che alle gravi materie, che nel fertile terreno di loro lingua vedevano allignare, e crescere, ed a maraviglia far prova; l'allegre, e piacevoli ad ora ad ora si tramischiassero, conoscendo bene; che per questo mezzo, più largo, ed ubertoso campo s'apriva loro, per cui con franco piede passeggiando, e diligentemente coltivandolo,

\footnotetext{
1. Le Prose fiorentine sono un florilegio di autori moderni degni di essere presi a modello di stile. Il frontespizio del volume primo (1661) recita «raccolte dallo Smarrito» (Carlo Roberto Dati), ma questo illustre accademico attese alla composizione e alla pubblicazione di quel solo primo volume. L'opera fu, in realtà, ripresa e continuata a partire dal 1716 da altri accademici e pubblicata in 17 volumi, divisi in tre parti, fino al 1745, compresa una ristampa del primo volume. La storia editoriale dell'opera è complicata da varie ristampe e riedizioni settecentesche, comprese due veneziane, per l'editore Occhi e per l'editore Remondini, nelle quali i volumi sono suddivisi in modo diverso.
} 
averebbe con tanta abbondevolezza risposto alle loro industriose fatiche, che ne averebbero potuto con agevolezza, di loro immortal linguaggio corre il più bel fiore in ogni diversa guisa di ragionare.

Nella cicalata, quindi, gli accademici mettevano alla prova la lingua fiorentina, dimostrando che essa era, o poteva diventare, uno strumento espressivo adatto ad «ogni diversa guisa di ragionare», per quanto bizzarra e capricciosa potesse essere. Per far questo, essi fecero confluire nella cicalata il livello più popolare della lingua, gli idiotismi locali (a volte allusivamente salaci, ma raramente volgari), i proverbi, gli aneddoti cittadini, insieme ad elencazioni varie, citazioni e pseudocitazioni, storpiamenti di parole; il tutto racchiuso in una sintassi sovrabbondante e argomentativa, che faceva largo uso di subordinate introdotte da che, di nessi relativi, di gerundive, anche premesse alla principale. Le parti sintatticamente complesse, inoltre, si alternavano a sezioni mimetiche del parlato, caratterizzate da frasi brevi e ricorso al discorso diretto, alle interiezioni, alle allocuzioni.

\section{Caratteri della cicalata}

Un chiaro esempio dell'andamento testuale di una cicalata è offerto dall'incipit della cicalata In lode della padella e della frittura di Lorenzo Panciatichi (Guasti, 1856: 21-24)2.

lo ogni sera all'osteria? Mi maraviglio di voi, Tengo casa aperta a Firenze: il mio cammino ha sempre la febbre col caldo; e per l'imbeccata snocciolo le monne di per dì: perchè noi non siamo più al tempo del Bugnola, che scriveva la carne, che dava a credenza, sul desco; e poi il venerdì, quando lo ripuliva, mandava il suo credito in raschiatura. Oggidì non si dà nè fa nulla per l'amor di Dio: e chi non lo sa, legga il comento di M. Mosco Lavaceci da Settimo, fatto sopra i Crepuscoli di Plutarco: e troverà, che facendosi un povero romito la barba per l'amor di Dio, il barbiere gli menava addosso il rasoio di tal foggia, che pareva che volesse arruotarlo sulle guance di quel povero uomo, facendo seco crudelmente a tacca. Ora si diede il caso, che nella bottega accanto un cane talmente abbaiava ed urlava, che pareva che avesse veduta la brutta Lou; onde il barbiere in valigia chiama il fattore, e gli dice: Guarda che diamine ha quel cane: nè volendosi il cane chetare, nè per pane nè per bastonate; e replicando il barbiere, Che diavol può egli avere? soggiunse quel povero romito, alzando le sue luci pietose: Egli averà forse qualcuno che gli fa la barba per l'amor di Dio. [...] O bene, o bene! Ora essendo io a tavola con un branco d'accademici, sgaraffando a luci torte le pagnotte, e sbaragliando le vivande con gli ugnoni, cominciammo a discorrere delle cose dell'Accademia, e particolarmente del Vocabolario della Crusca; nel qual proposito diceva uno de' nostri: Eh, io, in quanto a me, non credo mai a' tuoi distici poco significanti e mal concocenti, che fanno gran furie negli stimoli carnali; ma Piscia e va' a letto, diceva Salvino, che ordinava loro il rimedio.

2. La cicalata, del 1656, era stata raccolta, prima dell'edizione di Guasti (1856), anche in Prose fiorentine (1723: 189-215), con il titolo In lode della frittura. 
L'abbondanza di idiomi pone questo componimento in linea con alcune opere burlesche coeve, come le commedie di Michelangelo Buonarroti il Giovane (anch'egli, peraltro, autore di cicalate), ma soprattutto il Malmantile racquistato di Lorenzo Lippi. Alcuni idiotismi, significativamente, rimbalzano da una all'altra di queste opere, che evidentemente pescano in un patrimonio comune popolare e di letteratura giocosa. Ad esempio, essere in valigia 'essere adirato' si ritrova nella Fiera di Michelangelo Buonarroti il Giovane (II 3 11) e nel Malmantile racquistato (VII 63), e l'espressione colorita «sgaraffando a luci torte le pagnotte, e sbaragliando ${ }^{3}$ le vivande con gli ugnoni» deriva da un sonetto di Francesco Ruspoli, citato un po’ alla buona. Il componimento recita: «Ma se in casa altri batter può

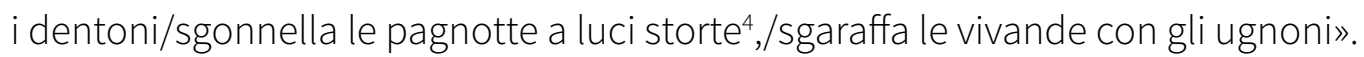

Le pseudoautorità citate, invece, sono prelevate di peso da un'altra cicalata, vero capostipite del genere, la Lezione o vero cicalamento di Maestro Bartolino dal canto de' Bischeri sopra 'I sonetto Passere e beccafichi magri arrosto ${ }^{5}$, di Giovanni Maria Cecchi (Cecchi: 1583/1868), del 1582, ma pubblicata nel 1583.

Tanto il ricorso alla tradizione di modi di dire e proverbi locali, quanto le scelte lessicali di Panciatichi rispecchiano un ideale linguistico che potremmo definire ipercruscante: la promozione della tradizione fiorentina contemporanea, quando possibile seguendo i classici, quando impossibile - perché il fiorentino contemporaneo divergeva dai classici-, ammettendo la lezione recente, trovando per essa giustificazioni in autori coevi o eventualmente nel Trecento minore. Tale ideale è più soggetto a compromessi nel Vocabolario, nel quale mancano alcune forme lessicali ed espressioni usate dagli autori di cicalate. In altri casi le forme appaiono per poi sparire in edizioni successive, a testimonianza della loro marcatezza diatopica.

Vediamo qualche esempio fono-morfologico che rimanda ad una fiorentinità ostentata nell'estratto di Panciatichi. Una forma rinascimentale squisitamente fiorentina è cammino 'camino', con raddoppiamento di nasale, sconosciuto prima del Cinquecento, ma registrato nella prima edizione del Vocabolario (e poi mantenuto fino all'ultima), accanto a camino.

Comento, con scempiamento della nasale protonica, sembrerebbe opporsi a cammino, a testimonianza di un progetto non sistematico, e invece è perfettamente in linea con l'ideale di lingua ipercruscante. A Firenze, infatti, si è sempre detto comento, a partire dal Trecento e fino a tempi relativamente recenti: la voce commento è usata una volta sola da Boccaccio nel Trattatello, ed è, per il resto, un'innovazione rinascimentale dovuta a prestito diretto dal latino, diffusa in ambito di koiné, in

3. L'edizione Prose fiorentine (1723: 192) riporta sgonnellando al posto di sbaragliando, forse una lectio facilior che avvicina la citazione all'originale del sonetto di Ruspoli.

4. Così legge l'edizione da me consultata (Ruspoli, 1876: 91), che, però, riporta in nota la variante torte.

5. Il sonetto, di Francesco Berni, è stato prima tradito con questo titolo. Ė citato così nel Vocabolario della Crusca fino alla IV edizione come esempio s.v. Beccafico. Nelle Giunte alla IV edizione del vocabolario (vol. 6, p. 43), però, i compilatori precisano: «BECCAFICO. Definiz: Bern. rim. agg. 1. 102. Passere, corr. Cancheri». Il titolo del sonetto è oggi (Berni, 1985: 6) stabilizzato come Sonetto contra la moglie; Cancheri, e beccafichi magri arrosto ne rappresenta il primo verso. 
autori come Niccolò da Correggio, Ortensio Lando, Tommaso Garzoni e Torquato Tasso. Ė entrata nel Vocabolario solamente nella quinta edizione, con la dizione «Commento e talora anche comento» (s.v. Commento) $)^{6}$.

Il futuro averà è meno marcato di cammino, ma è comunque una scelta a favore del moderno. Tra il Duecento e il Trecento, le forme non sincopate convivono con quelle sincopate (avr-), ma sono in larga minoranza. Le forme con dileguo della costrittiva (ar-), invece, sono pochissime, e soprattutto in autori non fiorentini. Nel Quattrocento, la situazione si capovolge: le forme con dileguo ${ }^{7}$ divengono le uniche o quasi usate a Firenze (in Leon Battista Alberti, Machiavelli, Lorenzo il Magnifico). Nel Cinquecento Bembo prescrive averò desinato (Prose, III, 47), mentre sfrutta anche le forme con sincope nelle Rime (una sola volta arà, una sola volta aranno), evidentemente per ragioni metriche. Varchi (1570/1846) ha esclusivamente le forme ar- (una sola occorrenza di avrö), che sono, evidentemente, ancora le più diffuse a Firenze ${ }^{8}$; dal canto suo, Muzio (1582/1846) ha 2 volte avrà, 2 volte averà, 1 averanno, mai l'altro tipo'. In generale, nel secolo XVI le forme sincopate sono in netta superiorità sulle altre due varianti. Tra queste ultime, quelle con dileguo sono maggioritarie e sono tanto fiorentine quanto "italiane": le troviamo anche in Castiglione (che usa in ugual misura il tipo aver-) e Trissino, per esempio.

Nel Seicento le tre forme continuano a convivere, con quelle sincopate ancora maggioritarie. Ma, se le forme non sincopate resistono, quelle con dileguo vanno scomparendo ${ }^{10}$ : la BIZ restituisce attestazioni numericamente significative per questo tipo solamente in due autori, fiorentineggianti: Michelangelo Buonarroti il Giovane (nella Tancia) e Galilei. Poche attestazioni sono anche in due autori settentrionali: nella Maria Maddalena peccatrice e convertita di Anton Giulio Brignole Sale e nel Brancaleone di Latrobio (Giovan Pietro Giussani). Galilei, Brignole Sale e Latrobio, peraltro, usano preferenzialmente le altre varianti: Galilei indifferentemente, Brignole Sale soprattutto quella sincopata, Latrobio quasi esclusivamente quella "piena". La situazione, insomma, vede, nel Seicento, la normalizzazione del tipo avr-, dentro e fuori Firenze, e la scomparsa del tipo ar- (tranne casi isolati, dovuti al gusto personale), ormai caratterizzato come tendenzialmente popolare o passatista. Nel momento in cui il tipo ar- è sentito non più come innovativo, ma semmai come conservativo, il tipo aver- rappresenta, per i cicalanti, una scelta almeno in controtendenza rispetto all'uso "italiano", che si sta orientando verso avr-.

6. Tutte le edizioni del Vocabolario sono state consultate nella versione on line, all'indirizzo www.lessicografia.it.

7. Il tipo arò, arei è indicato da Manni (1979: 141-142) come tratto caratterizzante del fiorentino quattrocentesco.

8. Approvate anche da Giambullari (cf. Marazzini, 1993: 168).

9. La preferenza di Muzio per le forme aver- è testimoniata da una citazione di un passo dell'Ercolano. L'originale è: «M. Lelio mio caro, desinato che aremo [Varchi (1570/1995: CCCLXXIX) ha haremo] e riposatici alquanto, potrete cominciare senza altre scuse o cirimonie» (Varchi 1570/1846: 15); la citazione: «M. Lelio mio caro, desinato che averemo, e riposatici alquanto, potrete cominciar senza altre scuse e cerimonie» (Muzio, 1582/1846: 654).

10. Sono, tra l'altro, condannate da Buommattei come fiorentinismi troppo marcati (cf. Marazzini, 1993: 191). 
Nell'introduzione alle Prose fiorentine (1723: V), sopra riportata, appaiono anche i condizionali averebbe e averebbero ${ }^{11}$, coerenti con il futuro averà, ma non del tutto scontati. Ritengo anche questa scelta protesa verso il fiorentino moderno. Innanzitutto, la desinenza -ebbero del condizionale III plurale è innovativa rispetto a -ebbono ${ }^{12}$. C'è da considerare, inoltre, la radice "piena". Le tre forme alternative del condizionale di avere, con e senza sincope e con dileguo della costrittiva (are-), convivono da Dante a Galilei. Boccaccio ha quasi sempre le forme con sincope, sporadicamente quelle con dileguo, quasi mai quelle "piene", ma Bembo (Prose, III, 47), coerentemente con il futuro averò desinato, prescrive proprio averei amato. Bembo stesso, tuttavia, preferisce nell'uso avr-: nelle Prose usa 8 volte avrebbe/avrebbono ${ }^{13}$, solamente 2 volte arebbe (in I, 14, in bocca a Lorenzo il Magnifico, che, nella sua produzione, ha sempre le forme are-, tranne due avre' per avrebbe), mai le varianti non sincopate. La BIZ restituisce, per il Cinquecento, 445 attestazioni di condizionale "pieno", 365 di quello con sincope, 966 di quello con dileguo (queste ultime sono le uniche usate da Varchi 1570/1846 ${ }^{14} \mathrm{e} \mathrm{di}$ gran lunga le preferite, ad esempio, da Anton Francesco Grazzini e Vasari). Le forme con dileguo, quindi, nel Cinquecento erano le più comuni, tipicamente fiorentine, ma in realtà panitaliane. Il Seicento vede una situazione completamente diversa: le forme con dileguo quasi spariscono, con appena 46 attestazioni in tutto il secolo. La distribuzione del condizionale con dileguo in quest'epoca è simile a quella del futuro ar-, si tratta di un uso idiosincratico di pochi autori, tra cui ancora Brignole Sale, Galilei, Buonarroti il Giovane, Latrobio. Per quanto riguarda le altre varianti, i numeri grezzi mostrano che quelle "piene" si affermano: la BIZ restituisce 412 attestazioni del tipo "pieno", 252 di quello con sincope. Un'analisi appena più attenta, però, mostra che il numero delle forme "piene" è gonfiato dall'uso altissimo di queste forme fatto da Paolo Sarpi: delle 412 attestazioni complessive, ben 284 vengono dalla Istoria del Concilio tridentino. Per avere un'idea più rispondente alla realtà, incrociamo i numeri assoluti con il numero degli autori che usano almeno una volta il condizionale con sincope: 19, con quello degli autori che usano almeno una volta il condizionale "pieno": 13. Alla luce di questi dati, possiamo, con buona approssimazione, concludere che il condizionale con sincope sia usato nel Seicento più frequentemente di quello "pieno". Quest'ultimo è, comunque, vivo nell'uso, tanto che tutti gli autori che lo usano sono anche nell'elenco di quelli che usano l'altro tipo ${ }^{15}$.

In conclusione, la scelta del condizionale "pieno" nell'introduzione è interpretabile come quella del futuro "pieno" all'interno della cicalata di Panciatichi: è la scelta più fiorentina e meno panitaliana possibile tra quelle presenti nell'uso del momento.

11. Averebbe è anche nella cicalata di Panciatichi (Guasti, 1856: 22).

12. «Ma ancora nel Seicento si ha la sensazione che il morfema sia minoritario tra gli scrittori non toscani» (Trovato, 1994: 193).

13. Celebre il passo: «Che quando e' [scil. Dante] disse: Biscazza, e fonde la sua facultate, Consuma o Disperde avrebbe detto, non Biscazza, voce del tutto dura e spiacevole; oltra che ella non è voce usata, e forse ancora non mai tocca dagli scrittori.» (Prose, II, 5).

14. Con l'eccezione di 1 avrei a p. 180 e 1 avrebbe a p. 472.

15. Nel Settecento la situazione si capovolge ancora, avvicinandosi all'uso attuale. La BIZ restituisce appena 54 attestazioni del tipo "pieno" (quasi tutte da commedie in italiano di Goldoni), 732 di quello con sincope (molte in Goldoni, ma molte anche in Metastasio, Alfieri e nella rivista /l Caffè), 20 del tipo con dileguo (17 delle quali in Giovan Battista Vico). 
Nella stessa introduzione appare il congiuntivo sieno ${ }^{16}$, che è un caso di convergenza tra antico e moderno. La forma con la vocale e è preferita (anche se convive con siano) tanto da Boccaccio quanto da Machiavelli. Bembo (Prose, III, 50) ammette parimenti sia, siano e sie, sieno - ma poi preferisce sia (favorito dal connettivo con ciò sia cosa che) e sieno. In tutto il Cinquecento la BIZ riporta 72 attestazioni di sie, 516 di sieno (soprattutto in fiorentini, toscani e sostenitori di Bembo, come Michelangelo, Pietro Aretino, Pietro Fortini e Ariosto). Sie sparisce del tutto nel Seicento, mentre sieno, sebbene largamente minoritario rispetto a siano, conta 126 attestazioni in tutto il secolo, non per forza in autori fiorentini o filofiorentini (anche in Bartoli, Boccalini, Campanella, Marino, Tassoni). Sieno, per i cicalanti, è, al pari del futuro e del condizionale "pieni" di avere, una forma orgogliosamente fiorentina, che lega passato e presente.

La forma debbe (presente indicativo), invece, è decisamente moderna. Boccaccio ha quasi sempre dee; Bembo (Prose, III, 29) ricorda che dee è in uso, ma che «Debbe, che la diritta voce è, dalle prose rifiutata, solo nel verso ha luogo, e Deve altresì. In realtà, debbe è quasi assente nelle Tre corone (e in generale usato sporadicamente nel Trecento) ${ }^{17}$ : di Boccaccio si è detto, Dante ha sempre dee e Petrarca preferisce deve (ha debbe una sola volta, forzato dalla rima con sarebbe; 2 volte dee). La forma con labiale intensa si afferma a partire dal Quattrocento: Leon Battista Alberti alterna dee e debbe; Machiavelli, per contro, ha quasi sempre debbe. Le tre forme, comunque, convivono anche a Firenze ancora nel Cinquecento, ma deve è decisamente la scelta meno fiorentina e più cortigiana: la troviamo in Castiglione, Equicola, Tasso, Trissino, nel Pedante di Belo e nell'Orlando furioso (giusta la concessione di Bembo). Varchi (1570/1846) ha deve 12 volte (debbe 22 volte, dee 43 volte). Tale scelta, per niente scontata, di deve, è criticata da Alessandro Tassoni, che in nota (Varchi, 1570/1846: 66) scrive: «Dee, e debbe disse il Bocc. et ogni buono Scrittore; essendo deve del verso» ${ }^{18}$. Nel Seicento deve è ormai diventato comune anche a Firenze, tanto che è la forma di gran lunga preferita da Galilei. A quell'epoca debbe doveva suonare come tipico, anche se non esclusivo, del fiorentino moderno: non si trova mai in Tesauro; Bartoli gli preferisce di gran lunga dee, anche se difende la forma:

Che questa parte al mio Signor si debbe: cosi scrisse l'Ariosto C. 3. st. I male, se ne crediamo al Ruscelli, che afferma indubitato, Debbe non essere il Deve, ò Dee rispondente al Debet, ma essere il Debuit de' Latini, e il Dovette italiano. Io non ho veduta questa sua osservatione a tempo di trovar piu esempi in quanto m’ho aperto innanzi il Crescenzi v'ho trovato lib. 3 c. 22. Toccarsi dal coltivator non si Debbe. che manifestamente suona Debet. (Bartoli, 1668: 415-416)

16. Sieno è anche in Salvini (1741: 218).

17. La forma è idiosincratica di Caterina da Siena, che nelle Lettere (Caterina da Siena 1973, in BIZ) la usa 60 volte.

18. Il curatore dell'edizione da me consultata, Pietro Dal Rio, chiosa ulteriormente la nota di Tassoni: «Così falsamente e pedantescamente postilla il Tassoni», mostrando quale era l'uso dominante a metà Ottocento (a favore di deve). 
Torniamo alla cicalata di Panciatichi. La forma arruotarlo è particolarmente interessante: è scritta così nelle edizioni Panciatichi (s.n.t. ma 1729: 7) e Guasti (1856: 22) della cicalata, mentre appare come arrotarlo nelle edizioni delle Prose fiorentine (1722?: 178) ${ }^{19}$, delle Prose fiorentine (1723: 190), delle Prose fiorentine (1730: 92), delle Prose fiorentine (1735: 92), delle Prose fiorentine (1751: 81). La forma con dittongo fuori di accento è un'innovazione estranea del tutto al Trecento, e quasi del tutto anche al Seicento; il lemma arruotare, però, appare accanto ad arrotare proprio nella terza edizione del Vocabolario (1691):

ARROTARE, e ARRUOTARE. Assottigliare il taglio de' ferri alla ruota. L. acuere, acutum reddere. Coll. S. Pad. Vedendolo il garzone fuor d'usanza arrotare il coltellaccio. Stor. Eur. 1. 18. Dove solo abbonda di ferro, e di pietre da arrotarlo. §. E per simil. neut. pass. Tac. Dav. Dial. El. 421. Pure s'arrotavano altramenti in quelle confusioni. §. Per metaf. Stropicciare, lisciare, Lat. expolire. Sag. Nat. Esp. 88. Ne anche a strofinare l'ambra in sul panno, avvegnachè arruotandovela su con gran forza, volle tirare. §. E Arrotarsi degli huomini, vale Agitarsi con inquietudine. Lat. anxie se exercere. Dav. Camb. 96. Quanto più gli huomini s'agitano, e s'inframmettono, e quasi s'arruotano insieme.

Il lemma era solamente arrotare nelle due edizioni precedenti e arruotare è eliminato già dalla quarta edizione. Curiosamente, l'eliminazione della variante con il dittongo fuori di accento comporta anche l'emendamento dell'unico esempio a favore riportato nella definizione, quello dei Saggi di naturali esperienze di Lorenzo Magalotti (1667: LXVIII), che, però, ha effettivamente il dittongo in quel luogo. Ė chiaro che arruotare è un idiotismo diffusosi a Firenze nella seconda metà del Seicento, accolto brevemente nel Vocabolario con il supporto di un'autorità non a caso contemporanea e non a caso accademica, ma poi abbandonato perché giudicato un occasionalismo. La forma marcata sparisce dalle edizioni settecentesche della cicalata di Panciatichi, curate dalla stessa Accademia della Crusca (e da quelle veneziane, che sono debitrici di quelle dell'Accademia), per gli stessi motivi che portano ad eliminarla dal Vocabolario, mentre viene mantenuta nelle edizioni esterne all'Accademia, Panciatichi (s.n.t. ma 1729), curata dal letterato Antonmaria Biscioni, e Guasti (1856), che da quella deriva.

19. Secondo Guasti (1856: LXVIII), questa edizione è successiva all'edizione del 1723, perché si ferma ai primi sei volumi e ha una suddivisione interna diversa rispetto all'edizione del 1723, che è, invece, completa. La data riportata nel frontespizio, 1722, potrebbe essere un errore per 1732, oppure un escamotage per godere delle stesse approvazioni ecclesiastiche e regie accordate all'edizione del 1723. 


\section{Contro la cicalata}

\subsection{Il Settecento}

Abbiamo visto grazie all'esempio autorevole di Lorenzo Panciatichi come l'ideale linguistico rispecchiato nelle cicalate sei-settecentesche fosse la promozione del fiorentino contemporaneo come lingua adatta ad ogni scopo. La natura apparentemente frivola e capricciosa di questi componimenti, insieme alla lingua ipercaratterizzata con cui erano scritti, però, comportò una lunga serie di reazioni molto critiche contro queste opere, non solo da parte dei tradizionali detrattori della Crusca.

Rispetto al Vocabolario, della cicalata molti illuministi settecenteschi sottolinearono la futilità degli argomenti. Giuseppe Baretti, fondatore e animatore della Frusta letteraria (1763-1765), cita le cicalate cruscanti in quattro occasioni nei circa due anni di vita della rivista ${ }^{20}$, mai riferendosi ad una in particolare ma considerandole come un corpus unico e monolitico. Nel medesimo corpus Baretti spesso inserisce anche la letteratura arcadica, macchiata, a suo dire, degli stessi peccati:

Ma vuoi tu dire, caparbio prete, che costà nella nostra metropoli sia così comunemente letto come lo sono le Ballerine onorate, i Bertoldi in rima, i Congressi di Citera, le Poesie degli Arcadi, le Cicalate de' Cruscanti, e tant'altre insulse filastrocche, dalle quali non v'è nulla da imparare, se non talora un qualche mal vezzo? (Baretti, 1763: 9)

Prodiga sei d'applausi ad ogni sciocco

che in Arcadia gesticola un sonetto

in lode dello sterco d'un allocco;

o t'ingalluzzi tutta nell'aspetto,

se un qualche perdigiorno ti regala

d'un marmo da più secoli negletto;

o ti metti sui nastri e sulla gala,

quando un qualche autoruzzo mosciolino

riboboleggia in cicalate e sciala;

o piangi perché morto è quel d'Urbino,

che non ti può somministrar disegni

pel libro di Bertoldo e Bertoldino. (Baretti, 1763: 90)

Nell'articolo "Risposta ad Aristofilo", nel numero 13 della rivista, del componimento accademico sono stigmatizzati l'astrusità delle pseudocitazioni e l'idiomaticità esasperata, giudicata popolare e tendente alla volgarità:

20. Nel calcolo considero un'unica occasione tre menzioni diverse, perché ruotanti intorno agli stessi giudizi: nella lettera in francese di Aristofilo (nel numero 13 della rivista), nella risposta alla lettera (nello stesso numero) e nella nota dell'autore (nel numero 18) contenente la risposta di Baretti a un tal Filologo Etrusco. 
Que' modi poi da voi notati nelle cicalate, e che a voi paiono enimmi e logogrifi, sono modi usati da' battilani, da' trecconi, da' pesciaiuoli, da' beccai, dalle sgualdrine, e da altra simil gente di Firenze e de' suoi contorni (Baretti, 1764: 190).

Molto colorita è la seguente tirata, nella quale le cicalate sono associate non alla maniera arcadica, ma alla tradizione classicista fiorentina, esemplificata da Boccaccio, Bembo e Della Casa:

Come mai un Genovesi ha potuto avvilirsi tanto da seguire i meschini voli terra terra di certi secchi e tisici uccellacci di Toscana? Eh, Genovesi mio, adopera gli abbindolati stili del Boccaccio, del Bembo e del Casa, quando ti verrà ghiribizzo di scrivere qualche accademica diceria, qualche cicalata, qualche insulsa tiritera al modo fiorentino antico o moderno; ma quando scrivi le tue sublimi meditazioni, lascia scorrere velocemente la penna; lascia che al nominativo vada dietro il suo bel verbo, e dietro al verbo l'accusativo senz'altri rabeschi; e lascia nelle Fiammette, e negli Asolani, e ne' Galatei, e in altri tali spregievolissimi libercoli, i tuoi tanti "conciossiacosacché", e i "perocché", e gl'"imperciocché", e i verbi in ultimo, el'"e" tra un addiettivo e l'altro, e il "confacentesi", e il "signoreggialo", e il "mancheranti", e il "Dio aiutantemi”, e tutte quell'altre cacherie e smorfie di lingua, che tanti nostri muffati grammaticuzzi vorrebbono tuttavia far credere il non plus ultra dello scrivere (Baretti, 1763: 19-20).

Baretti dimostra di non aver colto la grande differenza tra il classicismo bembiano e l'ideale linguistico degli accademici cicalanti: questi ultimi promuovevano il fiorentino contemporaneo, e lo facevano in modo oltranzistico, più di quanto avessero il coraggio di fare nel Vocabolario. D'altro canto, il critico della Frusta dichiara esplicitamente di disprezzare ugualmente il «modo fiorentino antico e moderno». Di quel modo porta ad esempio alcuni difetti esemplari (pescandoli dal brano che sta recensendo dalle Meditazioni filosofiche sulla Religione e sulla Morale dell'abate Antonio Genovesi), soprattutto sintattici, come congiunzioni composte e strutture frasali, e morfosintattici, come i participi presenti assoluti. Tra questi appare un solo appunto lessicale, quel signoreggialo invero mai usato nel libro (forse un refuso per signoreggiano, usato al pari di signoreggio e signoreggia).

La trattatistica scientifica ha ormai, in questa epoca, modelli italiani, come Galilei, Redi, Magalotti, Malpighi, e soprattutto europei, eppure alcuni autori ricorrono ancora a Bembo, Della Casa e persino alle concettose cicalate alla ricerca di modelli di lingua e di stile. Questi modelli, però, sono adatti alla prosa erudita di stampo scolastico, basata sulla logica aristotelica, non sull'esperimento; il genere della cicalata, che ridicolizzava l'ufficialità della lezione accademica esasperandone le caratteristiche linguistiche e stilistiche, finiva per essere ancora più incongruente con le nuove esigenze culturali dell'epoca: 
Il discorso scientifico, nella sua tendenza a tutelare un livello alto ma aperto al pubblico non specialista, si muove dunque tra lingua letteraria e lingua comune, pur cercando una sua specificità formale, legata ai nuovi canali di comunicazione, "giornali", "gazzette", "atti" di accademie, che impongono una scrittura essenziale, più "concentrata sugli aspetti euristici e tecnici della ricerca" [...]» (Matarrese, 1993: 74)

Baretti rappresenta proprio l'ideale illuministico dell'essenzialità, della semplicità, dell'espressione che segue il pensiero in modo naturale, della «pulitezza, e chiarezza di stile, che dimestica le materie ruvide e selvagge, che spiana le più ardue, che dilucida le più oscure» (Muratori, 1708: 120). La lingua "pulita" che Baretti propugna doveva assecondare le esigenze della prosa scientifica e filosofica, dare un nome alle nuove invenzioni, spiegare le scoperte della chimica, della medicina, della fisica. Si tratta di un ideale democratico, o almeno antiaristocratico, che collega lo svecchiamento dell'italiano scritto alla sua capacità di diffondersi a fasce più ampie della popolazione. Ma si tratta prima di tutto di un'idea di lingua, con i suoi modelli linguistici e culturali, le sue regole, i suoi ambiti d'uso.

Per tutto il Seicento, la cicalata è un tratto esclusivo della Crusca, dove era nata, e di qualche altra accademia fiorentina strettamente collegata alla Crusca, come quella degli Apatisti. Nel Settecento, invece, il genere si diffonde in tutta Italia, divenendo protagonista delle adunanze di molte accademie. La fortuna della cicalata è comprovata dalla menzione che ne fa Alessandro Verri nella celebre Rinunzia avanti il notaio, pubblicata sulla rivista /l Caffè (1764-1766):

In oltre considerando noi che le cose utili a sapersi son molte, e che la vita è breve, abbiamo consagrato il prezioso tempo all'acquisto delle idee, ponendo nel numero delle secondarie cognizioni la pura favella, del che siamo tanto lontani d'arrossirne, che ne facciamo amende honorable avanti a tutti gli amatori de' riboboli noiosissimi dell'infinitamente noioso Malmantile, i quali sparsi qua e la come gioielli nelle lombarde cicalate sono proprio il grottesco delle belle lettere. (Verri, Alessandro, 1766: 49)

Quali sono le lombarde cicalate tanto disprezzate da Verri? Ricordiamo che qualche cicalata accademica era stata composta tra gli anni Cinquanta e i Sessanta del secolo da Giorgio Giulini, Giuseppe Parini e Carl'Antonio Tanzi nell'ambito dei Trasformati milanesi (di cui fu membro in gioventù anche l'altro Verri, Pietro). Ma lombarde qui ricomprende probabilmente tutto il nord Italia, in un'ideale contrapposizione con la Firenze dei cruscanti21, e cicalate potrebbe indicare, per antonomasia, qualunque discorso, non per forza legato alle pratiche accademiche, intorno a temi linguistici; insomma potrebbe definire in una parola un certo modo di intendere la letteratura, la prosa, gli scopi e le funzioni della lingua.

21. L'interpretazione "allargata" del termine è supportata da Schweickard (2006: s. v. Lombardia): «ln origine Lombardia designa il territorio dell'Italia occupato dai Longobardi (568-773), dal sec. IX si riferisce per lo più a tutta l'Italia settentrionale». Significativamente, Schweickard cita, come fonte settecentesca, una lettera di un altro membro dei Trasformati milanesi, Giuseppe Baretti (da Baretti, 1839: 329): «Giuseppe [n.d.r.] non pare voglia spingere i pensieri tant'oltre; e noi [...] dobbiamo stare ginocchioni, pregando che cosi sia, e che non gli venga neppure la voglia di allargare i confini della Lombardia sua». 
Pietro Verri, nell'articolo / tre seccatori, ci fornisce un'ulteriore sfumatura del termine cicalata:

L'occupazione di scrivere, e singolarmente di scrivere un'opera periodica, pare molto geniale e graziosa, e certamente v'è qualche cosa che non è volgare nel piacere di vedersi in un regolato carteggio colla specie umana, [...] ottenere in somma l'approvazione di quei che più si stimano e qualche meschina cicalata da qualche rettile scrittore, contrassegni tutti di buon augurio. (Verri, Pietro, 1765/1766: 424)

Il termine è usato qui ancora in senso generico, a definire qualunque componimento d'occasione privo di spessore, interessato alla pura favella e non alle idee, non con riferimento al genere letterario inventato dai cruscanti. Sembra, inoltre, che la cicalata sia assunta come paradigma degli scritti polemici e delle invettive, liquidati come meschini.

Insomma, il termine cicalata per gli autori del Caffè ha dei contorni imprecisi: l'ideologia dell'utile che fa da sfondo alle opinioni linguistiche degli autori settecenteschi ha ridotto il genere campione dell'erudizione accademica ad uno stereotipo di ozio, di futilità, di polemica fine a sé stessa.

\subsection{L'Ottocento}

Un simile trattamento di sufficienza continua ad essere riservato al genere negli anni successivi. Nell'articolo Difesa dell'Opera seria, apparso, non firmato, nel Conciliatore (1818-1819) nel 1818, l'autore nomina en passant il genere come campione del discorso prolisso oltre il necessario, e lo associa allo stile latineggiante, dal periodo ampio e pieno di inversioni, ancora in voga nel Settecento; campione di questo stile sarebbe l'erudito Francesco Saverio Quadrio22.

Se ciò è vero, io vedo derivarne alcune gloriose conseguenze pel teatro italiano, le quali sviluppate colla necessaria prolissità potrebbero fare assai bene la loro figura in qualche bella CICALATA indiritta, per modo di dire, alla sempre felice memoria del molto reverendo e molto voluminoso padre Quadrio.

L'incompatibilità dichiarata tra l'autore dell'articolo e il tipo di scrittore alla Quadrio è linguistico-ideologica; riguarda, cioè, lo scopo che i due autori attribuiscono alla scrittura nei confronti della società. Quadrio è un modello ancora legato al bel discorso, alla pura favella già indicata da Alessandro Verri cinquant'anni prima come finalità da rigettare. La scrittura, secondo l'anonimo ottocentesco, che sia di argomento letterario o scientifico, che rifletta l'andamento del ragionamento o dell'esperimento, deve servire a trasmettere conoscenza al più ampio pubblico possibile, non a glorificare l'erudizione dell'autore. Il discorso deve essere, quindi, conciso e limitarsi a riportare le informazioni pertinenti, rifuggendo dai dettagli e da considerazioni accessorie.

22. Tra le opere di questo studioso spicca la colossale Della storia e della ragione d'ogni poesia (Milano, Agnelli, 1739-52), in sette volumi, ricca compilazione di generi, luoghi e nomi, inquadrati in una classificazione molto labile. 
Cicalata, comunque, non è del tutto ridotto a un termine antonomastico per la letteratura ritenuta oziosa; il genere rimane anche nell'Ottocento, persino tra gli autori del Conciliatore, riconoscibile, associato all'Accademia della Crusca e al classicismo filofiorentino. Nella recensione al volume Proposta di alcune correzioni ed aggiunte al Vocabolario della Crusca (Vol. II, Parte I) di Vincenzo Monti, la deriva plebea già disprezzata da Baretti nella Frusta letteraria è considerata ancora da Ludovico di Breme il trait d'union che lega Boccaccio, i canti carnascialeschi, Berni e le cicalate:

Diffatti se ci ha qualche altezza e disinvoltura d'idee, e nobiltà di discorso negli scrittori toscani ed altri di quelle età, simili doti le troviamo presso quelli che, spettatori del mondo intero, o del mondo ideale, trattarono argomenti storici, o epici o tragici; ma gli scrittori che somministrarono al vocabolario i più eletti fiori e i più reconditi modi; gli autori di cronache e cronachette, di novellieri di commedie, di cicalate, di carmi bernieschi e carnascialeschi gareggiarono in vece tutti di bassezza e di trivialità. II Boccaccio stesso, quanto non si mostrò egli inferiore ai due sommi che lo precedettero [scil. Dante e Petrarca], col non essersi saputo innalzare nè sopra la servitù del latinismo, nè sopra il fango, la materialità, e troppo spesso neppur sopra la goffaggine di quei costumi! (di Breme, 1819)

Se la linea editoriale del Conciliatore su quest'argomento è univoca, lo stesso non si può dire per la rivista Antologia (1821-1833), che alterna elogi e critiche. Una recensione del 1821, non firmata, alla nuova edizione del Porcus Trojanus ossia la Porchetta (Nardi, 1813/1821), accoglie l'operetta con moderato favore:

Così una scherzevole cicalata diventa una ricca miniera di notizie le meno comuni risguardanti a ciò che alla mense, alle vivande, alle cucine, ai cuochi appartiene, dottamente errando l'autore pei tempi antichi non solo, ma per gli eroici ancora raccogliendo sempre nuove dovizie di erudizione. (Anonimo, 1821: 174)

Non mancano, nella recensione, commenti positivi sul linguaggio:

E narra spezialmente i fasti dell'appetito, che'ei chiama eroico, con tal possesso de' classici tutti greci e latini, e di tutti gli scrittori d'archeologia, e con tanto vaga scelta di linguaggio, che più non di saprebbe desiderare.

A taluno però sembrar forse potrebbe a prima vista mancar talvolta la dettatura di naturalezza e di spontaneità, e ridondare a luoghi di voci e modi di dire meno usati dal comune degli scrittori, mentre in molti altri è disinvolta e naturale. Per es. e' dice: "possa io essere orticheggiato e ramatato se fra centinaia di migliaia si trovano due o tre buone raccolte (poetiche). II resto ghierabaldane che danno del macco a josa, pantraccole da rebechino". Ma in una sua nota si protesta che "quanto a parecchi vocaboli da me usati nel testo e nelle note, niuno attribuisca una certa ridondanza de' medesimi ad affettazione, ma a semplice scherzo che ben conviensi alla cicalata: del resto io spero di non averne usati a bizzeffe". (Anonimo, 1821: 174) 
La prima edizione di Nardi (1813/1821), in realtà, aveva ricevuto una recensione ancora più laudatoria, sulla rivista /l Poligrafo, a firma B.:

Spezialmente poi si è proposto l'Autore di sfoggiare non solo tutta l'erudizione che in molte centinaja di Scrittori è sparsa sopra l'oggetto suo, ma di fare inoltre vaga, e ricca pompa di sceltissimo linguaggio nostro, cercando e usando que' vocaboli che nel tempo stesso sono bellissimi, autentici, e poco adoperati [...]: mentre protestiamo, che sebbene il nostro A., forse a bella posta, eccede, pure crediamo giovevolissimo, come piacevolissimo troviamo, lo scoprimento e l'uso di una grandissima parte della tanto doviziosa nostra favella, nella qual parte appunto, ordinariamente ignorata, stanno e vezzi e grazie e forza più di quanto mai moltissimi scrittori italiani neppur si figurano. (B., 1813: 477)

Gli autori delle due recensioni assolvono l'erudizione prolissa, l'argomento futile, le espressioni fantasiose e bizzarre, che altri avevano segnalato come i difetti peggiori del genere. Ma c'è di più: questi autori ottocenteschi, uno probabilmente fiorentino, l'altro probabilmente milanese, elogiano proprio il lessico raro ed espressionistico del Porcus trojanus, che è, si badi, fortemente connotato come fiorentino cinquecentesco burlesco. Limitiamoci a ghierabaldane, pantraccole e rebechino: il primo termine è assente nella BIZ, mentre è lemmatizzato nel Vocabolario nella terza e nella quarta edizione, accanto alle varianti ghiarabaldana e ghiabaldana (nella quarta anche ghiabaldano), con il significato di 'Cosa di nullo pregio' (s. v. ghierabaldana). Il passo portato a testimone di questa parola è dalle rime di Alessandro Allegri, poeta satirico e burlesco vissuto a Firenze a cavallo tra il Cinquecento e il Seicento, tra l'altro fondatore dell'Accademia della Borra. Pantroccola, assente nella BIZ, appare nel Vocabolario solamente nella quarta edizione, con il significato di 'fola' e una testimonianza dalla Fiera di Michelangelo Buonarroti il Giovane. Rebechino, che in realtà è rabacchino, assente nella BIZ, appare solamente nella terza edizione (non più nella quarta) del Vocabolario, come 'Dim. di Rabacchio [moccioso n.d.r.]' e con una testimonianza dalla commedia /l furto di Francesco D’Ambra, del $1544^{23}$. Per quanto riguarda orticheggiare e ramatare, il primo verbo è attestato nella BIZ in Sacchetti e nel Falstaff di Verdi. Sacchetti è la stessa autorità citata dal Vocabolario, che lemmatizza il verbo già nella prima edizione e non lo espunge mai: solamente nella quinta edizione aggiunge alla definizione «Voce foggiata per ischerzo». Il secondo verbo è attestato a partire dalle commedie di Michelangelo Buonarroti il Giovane, anche se i nomi ramata e ramatata sono usati, sporadicamente, dal Quattrocento (Machiavelli, Doni, Ramusio). Proprio le commedie di Buonarroti sono portate come autorità dal Vocabolario, che lemmatizza il verbo solamente nella quarta edizione.

All'opposto, non lascia dubbi sul proprio giudizio negativo sulle cicalate sei-settecentesche Pietro Giordani, che, in una lettera a Gino Capponi pubblicata su Antologia commenta il valore letterario delle Prose Fiorentine, soffermandosi anche su quello specifico dei volumi di materie scherzose e piacevoli:

23. Questo autore entrò a far parte dell'Accademia degli Umidi, poi Fiorentina, già nel 1541, e nel 1551 fu eletto riformatore della lingua toscana in una commissione di cinque membri che comprendeva Varchi e Giambullari. 
quale umana pazienza basterebbe a sostenere i due volumi di materie scherzose e piacevoli? scherzose? sien pure: ma piacevoli, dio buono, quelle 27 cicalate! Dacchè il genere umano imparò a scrivere, si vide mai più strano abuso di parole e di tempo? In quale altra nazione entrò mai tale delirio? (Giordani, 1825: XX)

Vale la pena ricordare che in una raccolta di prose del 1855, Pietro Fanfani riporta la lettera di Giordani e chiosa in nota il giudizio dato trent'anni prima dall'intellettuale illuminista, ricordando almeno il merito lessicografico delle cicalate:

Le Cicalate, chi le considera per la sola materia che trattano, sono, è vero, cose frivole e baggianate: ma chi pensi che erano scritte col proposito di accozzare insieme i modi proverbiali e i proverbj fiorentini, e i più efficaci parlari popolareschi, vedrà di quanto profitto possono riuscire agli studiosi della lingua. Che poi non si salvasse dal far Cicalate la profonda testa del Bellini, e neppure, dirò io, la profondissima del Rucellai, e la quadratissima del Salvini, ec. non darà maraviglia a chi guardi in che occasione le Cicalate si facevano: ed a chi non crede che i sapienti debbano essere sempre musoni e intronizzati, nè pigliar giammai un po' di scianto, e ridere e sollazzarsi. Narratur et prisci Catonis Saepe mero coluisse virtus, e Semel in anno licet insanire. (Fanfani, 1855: 54 n.1)

Si vede qui la differenza tra il giudizio militante dell'intellettuale interessato alla funzione sociale della lingua e all'impatto culturale della letteratura, e la valutazione retrospettiva, distaccata, dello studioso di storia della letteratura.

Tanto il confronto tra Giordani e Fanfani, quanto il confronto tra le recensioni a Nardi (1813/1821) e la lettera di Giordani mostrano che la condanna delle cicalate non è totale: anche nell'ambito dell'illuminismo più maturo rimane in alcuni autori il gusto per l'erudizione "inutile" (spesso persino fasulla), per l'inventiva lessicale e per l'espressività tipica di queste opere.

La questione, peraltro, è ancora più complessa, come complesso è il quadro culturale dell'epoca: appena quattro anni dopo la lettera di Giordani, in una temperie sociale e culturale nella quale il Romanticismo ha preso piede e sta modificando i parametri culturali dell'illuminismo, Un italiano (il ventiquattrenne Giuseppe Mazzini) così difende, sulle pagine della stessa Antologia, la tradizione dei popoli (e quella italiana):

Così la Letteratura Italiana ebbe ne' suoi principi la impronta del Gusto che gli arabi aveano comunicato al mezzodi dell'Europa: fu platonica, mistica, e tendente all'idealismo in un secolo, inchinò al materialismo in un altro: severa e nazionale in un tempo, suonò parole d'indipendenza, e di magnanimo sdegno: imitatrice servile in un altro, fu inetta e lasciva, trastullo a' fiacchi, adulazione ai potenti: e il cielo italico diffondeva l'incanto dell'eterno sorriso nell'anima dei trovatori, come in quella di Guinicelli; all'epoca di Dante, come a quella delle cicalate» (Mazzini, 1829: 95-96). 
Mazzini, per sostenere le radici europee della tradizione italiana, individua una catena di modelli letterari alternativa a quella dei detrattori delle cicalate: la sequenza Boccaccio, canti carnascialeschi, Berni, cicalate, Arcadia è sostituita da quella arabi, trovatori, Guinizelli, «epoca di Dante» (quindi Dolce stil novo), cicalate. La possibilità di associare con tanta variabilità la cicalata ad esperienze letterarie così diverse, ma anche il giudizio stesso su quelle esperienze letterarie, che giustifica l'associazione con la cicalata, mostra, se ce ne fosse bisogno, che la questione è ideologica, e dipende dall'intento dei critici di promuovere una certa visione, a loro cara, della società, del ruolo della letteratura, della funzione della lingua, fondandola su una certa interpretazione, necessariamente parziale, della storia della lingua e della storia della letteratura ${ }^{24}$.

La voce romantica di Mazzini è, comunque, isolata: la cicalata cruscante è sentita generalmente come un esempio, letterario e linguistico, inadatto ai tempi. Al coro dei detrattori senza riserve si unisce anche il giornalista e fondatore di riviste Edoardo Scarfoglio ${ }^{25}$, nel volume // Libro di Don Chisciotte:

Dove sono andate a finire tutte quelle classi di letterati, dilettanti o mestieranti, che pur giovavano, se non altro, alla vendita dei libri? Dov'è l'abate, professore di belle lettere, autore di un trattato intorno all'arte dello scrivere? Dov'è il canonico, autore d'un mese di Maria in versi sciolti e d'una versione in terza rima del Salterio? E l'academico tronfio d'una cicalata sul miglior modo di tostare il caffè, recitata in concistoro? E il parassita rimatore, che in due giorni derivava dalla facile vena un sonetto per monsignore arcivescovo e una canzone petrarchesca per le nozze della duchessina e un madrigale pel ventaglio della principessa e una inscrizione per la tomba del pizzicagnolo, strappando la vita a morso a morso, a furia di endecasillabi? E i dilettanti di piccola erudizione, e i questionatori grammaticali, e gli armenti di Arcadia, dove dunque sono andati a finire? La stampa periodica, politica o letteraria, quotidiana o domenicale o bimensile o mensile, li ha tutti assorbiti. Chi riconoscerebbe in Ferdinando Martini il presidente dell'Accademia dei Tribolati; e chi nel Rigutini uno dei tanti che intorno al Perticari al padre Cesari a Basilio Puoti strillavano o predicavano teoriche linguistiche, e davano al minuto lezioni di purismo? Nessuno, certo; poichè anche le ultime pastorelle arcadiche stampano versi nei giornali clericali, o, al più, compilano diari danteschi. (Scarfoglio, 1885: 208-209)

\footnotetext{
24. Da non sottovalutare, nella concatenazione immaginata dal giovane Mazzini, il valore geografico, quindi politico-territoriale, delle esperienze letterarie menzionate: i trovatori rimandano all'Italia nord-occidentale, Guinizelli alla regione padana, Dante e il Dolce stil novo a Firenze. In questo quadro, le cicalate, genere letterario nato municipale, ma all'epoca dell'articolo ormai praticato - non solo letto - dalle Alpi all'Etna, potrebbero rappresentare per Mazzini una conferma dell'esistenza di uno spirito italiano; uno spirito non lirico o tragico, bensì comico.
}

25. Collaboratore, tra le altre, delle riviste Capitan Fracassa, Cronaca bizantina dell'editore Angelo Sommaruga, Domenica letteraria, fu fondatore del Corriere di Roma, del Corriere di Napoli, del Mattino. 
Più di un secolo dopo Giuseppe Baretti, Scarfoglio sfodera un immaginario letterario nel quale si muovono, seppur mutatis mutandis, gli stessi fantasmi aleggianti nella Frusta letteraria: l'academico tronfio che realizza cicalate d'occasione è associato ai poeti del petrarchismo arcadico ormai ridotto a una compilazione di stilemi, nonché ai grammatici, campioni dei quali sono adesso i puristi Cesari e Puoti. In effetti, nel 1885 la tradizione lirica che affondava le radici nel remoto Trecento era morente, ma non morta; la questione della lingua, inoltre, doveva ancora registrare interventi fondamentali, e vedeva contrapposti per il momento il culto del Trecento e la ricerca di una norma moderna. In direzione di quest'ultimo ideale operavano i giornali, protagonisti dell'evoluzione linguistica lungo tutto il secolo. Essi proponevano modelli sempre più snelli e lessicalmente aggiornati:

lo svecchiamento della prosa tradizionale operato dal Manzoni con la revisione dei Promessi Sposi [...] procede di pari passo con l'invenzione di uno «stile giornalistico» che rinunci a termini peregrini, ricorra a una sintassi prevalentemente coordinativa, povera di inversioni e di tmesi, si rivolga al lettore non specializzato assumendosi il compito di una seria divulgazione. (Serianni, 1989: 34-35)

È una testimonianza giornalistica, già tredici anni prima del libro di Scarfoglio, a mostrare che si è completato il processo di detecnicizzazione del termine cicalata. Un curioso giornale trisettimanale romano, il Don Pirloncino, pubblica nel 1872 un trafiletto dal titolo "Cicalate estive", a firma di un certo Ghiri... (i quattro puntini fanno parte della firma, probabilmente in sostituzione del completamento della parola: -gori). Il trafiletto contiene una serie di commenti ironici e leggeri ad alcune notizie di costume e di cronaca. Ad esempio:

Un giornale americano termina così una notizia necro-biografica. Per la morte di quest'uomo, la Società perde uno de' suoi più belli [sic] ornamenti, la Chiesa un fedele, sua moglie un marito costante, e noi un abbonato regolarissimo nei pagamenti.

Tat [sic] is the question!... (Ghiri... 1872)

Nell'epoca in cui nasce lo «stile giornalistico», le cicalate accademiche sono divenute oggetti del passato. Di esse è rimasta solamente un'eco nel termine cicalata, divenuto sinonimo di 'osservazione scherzosa, inezia'26.

26. Nella seconda metà del secolo, le pubblicazioni che si presentano come cicalate sono quasi sparite. E anche quando si presentano come tali, non sempre lo sono nel senso accademico: è il caso delle Cicalate polemiche intorno alle moderne difese degli antichi errori nell'insegnamento delle Matematiche di Sebastiano Purgotti (Perugia, Bartelli, 1871). Il titolo dell'opera, serissima e del tutto estranea a questioni linguistiche, è così spiegato dallo stesso autore: «A queste mie osservazioni appunto perché senza ordine e nesso ho dato il titolo di cicalate» (ivi: 80). 


\section{Bibliografia}

Anonimo (1953-1965) [1818], «Difesa dell'Opera seria», /l Conciliatore, a cura di Vittore Branca, Firenze, Le Monnier, in BIZ.

Anonimo (1821), «Porcus Trojanus ossia la Porchetta; Cicalata nelle nozze di M. Carlo Ridolfi Veronese con Madonna Rosa Spina Riminese: altra edizione, da' Tipi Nobili 1821 (Bologna) in 8.», Antologia, tomo IV, n’X (ottobre), p. 173-176.

B. (1813), «Porcus Trojanus, o sia la Porchetta, Cicalata ne le nozze di Messer Carlo Ridolfi con Madonna Rosa Spina. Arimino dai Tipi Albertiniani 1813», /l Poligrafo, anno III, n³0, p. 477-479.

Baretti, Giuseppe (Aristarco Scannabue) (1763-1765), La Frusta letteraria, tomi primo (n I-XII) e secondo ( $n^{\circ}$ XIII-XXV) (1763-1764), Roveredo, s.n. (Venezia, tip. Antonio Zatta); tomo terzo (da n XXVI a $\left.n^{\circ} X X X I I I\right)(1765)$, s.l. (Ancona), s.n.

Baretti, Giuseppe (1839), Lettere familiari di G. B. a'suoi tre fratelli Filippo, Giovanni e Amedeo, Milano, Società Tipografica de' Classici Italiani.

Bartoli, Daniello (1668), Il torto e 'I diritto del non si può dato in giudicio sopra molte regole della lingua italiana esaminato da Ferrante Longobardi, Roma, Varese.

Bembo, Pietro (1966), Prose e rime di Pietro Bembo (ed. Carlo Dionisotti), Torino, UTET, in BIZ.

Berni (1985), Rime, ed. Danilo Romei, Milano, Mursia, in BIZ.

BIZ, Biblioteca italiana Zanichelli, DVD-ROM (ed. Pasquale Stoppelli), Bologna, Zanichelli, 2010.

Caterina da Siena (1973), Lettere, ed. Gabriella Anodal, Roma, Edizione del Centro Nazionale di Studi Cateriniani, in BIZ.

di Breme, Ludovico (1819), «Recensione alla "Proposta di alcune correzioni ed aggiunte al Vocabolario della Crusca”», Il Conciliatore (ed. Vittore Branca), Firenze, Le Monnier, in BIZ.

Cecchi, Giovanni Maria (1868), Lezione o vero cicalamento di Maestro Bartolino dal canto de' Bischeri sopra 'I sonetto passere e beccafichi magri arrosto, Bologna, Romagnoli [1 $1^{\text {a }}$ ed.1583, Firenze, Manzani].

Fanfani, Pietro (1855), Lettere precettive di eccellenti scrittori, Firenze, Barbera, Bianchi e comp.

Giordani, Pietro (1825), «D’una scelta di Prosatori Italiani. Pietro Giordani a Gino Capponi Marchese», Antologia, tomo XVII (gennaio, febbraio, marzo), p. V-XXVII.

Guasti, Cesare (1856), Scritti vari di Lorenzo Panciatichi, accademico della Crusca, Firenze, Le Monnier.

Magalotti, Lorenzo (1667), Saggi di naturali esperienze, Firenze, Cocchini all'insegna della Stella.

Manni, Paola (1979), «Ricerche sui tratti fonetici e morfologici del fiorentino quattrocentesco», Studi di grammatica italiana, n० 8, p. 115-171. 
Marazzini, Claudio (1993), Il secondo Cinquecento e il Seicento, Bologna, il Mulino.

Matarrese, Tina (1993), /l Settecento, Bologna, il Mulino.

Mazzini, Giuseppe (un italiano) (1829), «D’una letteratura europea», Antologia, XXXVI, n 107 (novembre), p. 91-120.

Muratori, Ludovico Antonio (Lamindo Pritanio) (1708), Riflessioni sopra il buon gusto intorno le scienze e le arti, Venezia, Pavino.

Muzio, Girolamo (1846), La Varchina, in Varchi (1846), p. 649-750 [1ª ed. 1582, Venezia, Dusinelli].

Nardi, Luigi (1821), Porcus Trojanus ossia la Porchetta; Cicalata nelle nozze di M. Carlo Ridolfi Veronese con Madonna Rosa Spina Riminese, Bologna, Nobili [1ª ed. 1813, Arimino, tipi Albertiniani].

Panciatichi, Lorenzo (s.n.t. ma 1729), Cicalate di Lorenzo Panciatichi, eredi di Gabriello Tournes (ed. Antonmaria Biscioni, Firenze, Nestenus e Moücke).

Prose fiorentine (1661), Prose fiorentine raccolte dallo Smarrito accademico della Crusca. Parte prima contenente Orazioni. Volume primo, Firenze, Nuova Stamperia all'insegna della Stella.

Prose fiorentine (1722?), Raccolta di prose fiorentine, parte terza, volume primo, contenente cose giocose, Firenze, Tartini e Franchi.

Prose fiorentine (1723), Raccolta di prose fiorentine, parte prima, volume sesto, contenente cicalate, Firenze, Tartini e Franchi.

Prose fiorentine (1730), Prose fiorentine raccolte dallo Smarrito accademico della Crusca. Tomo secondo contenente orazioni, e cicalate, Venezia, Occhi.

Prose fiorentine (1735), Prose fiorentine raccolte dallo Smarrito accademico della Crusca. Tomo secondo contenente orazioni, e cicalate, Venezia, Occhi.

Prose fiorentine (1741), Raccolta di prose fiorentine, parte terza, volume secondo, contenente cose giocose, Firenze, Tartini e Franchi.

Prose fiorentine (1751), Raccolta di prose fiorentine, parte terza, volume primo, contenente cose giocose, Venezia, Remondini.

Ruspoli, Francesco (1876), Sonetti di Francesco Ruspoli editi ed inediti col commento di Andrea Cavalcanti non mai fin qui stampato, Bologna, Romagnoli.

Salvini, Anton Maria (1734), Prose Toscane, vol. I, Venezia, Pasinelli [1 $1^{\text {a }}$ ed. 1715, Firenze, Guiducci e Franchi].

Salvini, Anton Maria (1741), Cicalata duodecima In lode della Cicala, in Prose fiorentine, p. 212-238.

Scarfoglio, Edoardo (1885), Il libro di Don Chisciotte, Roma, Sommaruga.

Schweickard, Wolfgang (2006), Deonomasticon Italicum. Volume II. Derivati da nomi geografici: F-L, Tübingen, Niemeyer. 
Serianni, Luca (1989), Il primo Ottocento, Bologna, il Mulino.

Trovato, Paolo (1994), Il primo Cinquecento, Bologna, il Mulino.

Varchi, Benedetto (1846), L'Ercolano. Dialogo di Benedetto Varchi dove si ragiona delle lingue e in particolare della toscana e fiorentina con la correzione di Lodovico Castelvetro e la Varchina di Jeronimo Muzio, con le note di G. Bottari e di G. A. Volpi, Firenze, Agenzia libraria [1ª ed. 1570, Ercolano, dialogo nel qual si ragiona generalmente delle lingue e in particolare della fiorentina e della toscana, Giunti, Firenze].

Varchi, Benedetto (1995), L'Hercolano. Dialogo di Messer Benedetto Varchi (ed. Antonio Sorella), Pescara, Libreria dell'Università, 2 voll. [1ª ed. 1570, Ercolano, dialogo nel qual si ragiona generalmente delle lingue e in particolare della fiorentina e della toscana, Giunti, Firenze].

Verri, Alessandro (1766), «Rinunzia avanti il Notaio degli Autori del presente Foglio periodico al Vocabolario della Crusca», Il Caffè, tomo I, p. 47-50.

Verri, Pietro (1766), «l tre seccatori», // Caffè, tomo I, p. 424-430. 